\title{
MAXIMAL IDEALS IN TENSOR PRODUCTS OF BANACH ALGEBRAS
}

\author{
BY ARNOLD LEBOW ${ }^{1}$
}

Communicated by B. Yood, March 18, 1968

In this note we characterize the maximal left ideals in certain tensor products of complex Banach algebras. Although our methods are different, the results presented here resemble results of Gelbaum $[1],[2]$ that characterize the maximal two-sided ideals in the greatest cross norm tensor product of Banach algebras.

Let $A$ be a commutative Banach algebra with identity 1 , and let $B$ be an arbitrary Banach algebra with identity $e$. It follows from the universal property $[3, \mathrm{p} .181]$ of the algebraic tensor product $A \otimes B$ that if $M$ is a maximal ideal of $A$, then $M$ induces a homomorphism $h_{M}$ of $A \otimes B$ onto $B$ by the formula

$$
h_{M} \sum a_{i} \otimes b_{i}=\sum a_{i}(M) b_{i} .
$$

In all that follows we denote by $a$ the completion of $A \otimes B$ in some cross norm such that each homomorphism $h_{M}$ is bounded, hence has a unique extension to $a$. The greatest cross norm has this property. When $A$ is semisimple, another cross norm with this property is the sup norm

$$
\left\|\sum a_{i} \otimes b_{i}\right\|=\sup \left\|\sum a_{i}(M) b_{i}\right\| .
$$

With these conventions understood the main result can be stated as follows.

Theorem. $A$ subset $\mathcal{L}$ of $Q$ is a maximal left ideal if and only if $A$ contains a maximal ideal $M$ and $B$ contains a maximal left ideal $L$ such that $\mathscr{L}=h_{M}^{-1}(L)$.

Proof. To prove the sufficiency, let us suppose $\&$ has the desired form and that $\mathscr{L}^{\prime}$ is a left ideal which properly contains $\mathscr{L}$. Because $h_{M}$ is onto $B, h_{M}\left(\mathcal{L}^{\prime}\right)$ is a left ideal which properly contains $L$. Since $L$ is maximal in $B$, it follows that there is an element $x$ in $\mathcal{L}^{\prime}$ such that $h_{M}(x)=e$. Now $x-1 \otimes e$ belongs to the kernel of $h_{M}$ which is contained in $\mathscr{L}^{\prime}$, thus $1 \otimes e$ belongs to $\mathscr{L}^{\prime}$ and so $\mathscr{L}^{\prime}=Q$, proving that $\mathscr{L}$ is maximal.

${ }^{1}$ Supported in part by NSF Grant GP-5436. The author is indebted to the principal investigator of this grant, Professor B. R. Gelbaum, for several informative conversations. 
Now let $\mathscr{L}$ be a maximal left ideal in $a$. The kernel $K$ of the left regular representation of $a$ on the difference space $a-\mathcal{L}$ is a primitive ideal. Let $\pi$ denote the canonical map of $a$ onto the quotient algebra $Q / \mathcal{K}$. Since $B$ has an identity, $A$ may be imbedded in $Q$ and we denote by $\pi_{A}$ the restriction of $\pi$ to the subalgebra $A$. The kernel of $\pi_{A}$ is $A \cap \nVdash$ and it follows that $A / A \cap \nVdash$ is isomorphic to a subalgebra of the center of the primitive algebra $Q / \mathcal{K}$. But since the center of a primitive algebra with identity is isomorphic to the complex numbers $[4$, p. 61], it follows that $A / A \cap \Re$ is isomorphic to the complex field, and so $A \cap \nVdash$ is a maximal ideal $M$ of the algebra $A$. It is easy to verify that $\pi=\pi_{B} \circ h_{M}$ so that $\nVdash$ contains the kernel of $h_{M}$. But by definition $\mathscr{L}$ contains $\mathscr{K}$ and thus $\&$ contains the kernel of $h_{M}$. Using the same argument as in the proof of the sufficiency, it can be seen that $h_{M}(\mathscr{L})$ is a proper left ideal in $B$, hence there is a maximal left ideal $L$ containing $h_{M}(\mathscr{L})$. Therefore, $\mathscr{L}$ is contained in the maximal left ideal $h_{M}^{-1}(L)$, however $\&$ was assumed maximal, so it follows that $\mathfrak{L}=h_{M}^{-1}(L)$, completing the proof.

The need for this result was first suggested to us in the study of certain problems concerning functions whose values are Fredholm operators. We hope to complete that study in a future paper. For the present, we conclude with some of the applications of the theorem.

Recall that the intersection of the maximal left ideals is the radical of an algebra. An element $x$ in $Q$ is in the radical of $a$ if and only if $h_{M}(x)$ is in $L$ for each maximal ideal $M$ of $A$ and each maximal left ideal $L$ of $B$.

Corollary 1. The tensor product $a$ is semisimple if and only if $A$ and $B$ are semisimple.

An element of any algebra is left invertible if and only if it does not belong to any maximal left ideal.

Corollary 2. An element $x$ of $a$ is left invertible if and only if $h_{M}(x)$ is left invertible for each maximal ideal $M$.

We denote by $\sigma_{l}(x)$ the left spectrum of an element $x$ of an algebra.

Corollary 3. If $x$ is in $a$, then $\sigma_{l}(x)=\bigcup \sigma_{l}\left(h_{M}(x)\right)$. In particular, $\sigma_{l}(a \otimes b)=\sigma(a) \cdot \sigma_{l}(b)$.

Corollary 4. If $x_{1}, \cdots, x_{n}$ are elements of a such that for each maximal ideal $M$ of $A$ there exist elements $b_{1}, \cdots, b_{n}$ of $B$ such that $b_{1} h_{M}\left(x_{1}\right)+\cdots+b_{n} h_{M}\left(x_{n}\right)=e$, then there exist elements $y_{1}, \cdots, y_{n}$ of a such that $y_{1} x_{1}+\cdots+y_{n} x_{n}=1 \otimes e$. 


\title{
REFERENCES
} 297-310.

1. B. R. Gelbaum, Tensor products of Banach algebras, Canad. J. Math. 11 (1959),

2. - Note on the tensor product of Banach algebras, Proc. Amer. Math. Soc. 12 (1961), 750-757.

3. S. Mac Lane, Homology, Springer-Verlag, Berlin, 1963.

4. C. E. Rickart, Banach algebras, Van Nostrand, New York, 1960.

University of California, Irvine

\section{ON EIGENVALUE DISTRIBUTIONS FOR ELLIPTIC OPERATORS WITHOUT SMOOTH COEFFICIENTS. II}

\author{
BY RICHARD BEALS ${ }^{1}$
}

Communicated by Gian-Carlo Rota, April 5, 1968

In two previous papers the known asymptotic formula for the eigenvalues of a selfadjoint elliptic boundary value problem was extended to some cases of operators without smooth coefficients: to the Dirichlet problem in [1] and to the general coercive differential boundary value problem in [2]. The object of this note is to complete this study by proving the formula for general (i.e. not necessarily differential) boundary value problems on domains without smooth boundary. We use the methods of [1], [2]. It should be noted that the case of differential boundary conditions can be handled in a different way; see [3].

Let $\Omega$ be a bounded open set in $R^{n}$ with boundary $\partial \Omega$ which is regular in the sense of Calderón [4], i.e. satisfying the "restricted cone condition." Let $\boldsymbol{A}=\sum a_{\alpha}(x) D^{\alpha}$ be an operator defined on $\Omega$, with coefficients in $L^{\infty}(\Omega)$ and top-order coefficients uniformly continuous on $\Omega$. We assume that $\boldsymbol{A}$ is formally selfadjoint and uniformly elliptic of order $m$. Let $A$ be a selfadjoint realization of $\boldsymbol{A}$ in $L^{2}(\Omega)$, with domain $D(A) \subset H^{m}(\Omega)$. Set

$$
c(A)=(2 \pi)^{-n} \int_{\Omega} \int_{a(x, \xi)<1} d \xi d x,
$$

\footnotetext{
${ }^{1}$ Research partially supported by a contract with the Army Research OfficeDurham.
} 